\title{
Subaru optical observations of the old pulsar PSR B0950+08
}

\author{
S. V. Zharikov ${ }^{1}$, Yu. A. Shibanov², A. B. Koptsevich ${ }^{2}$, N. Kawai ${ }^{3,4}$, Y. Urata ${ }^{3,4}$, V. N. Komarova ${ }^{5,6}$, V. V. Sokolov ${ }^{5,6}$, \\ S. Shibata ${ }^{7}$, and N. Shibazaki ${ }^{8}$
}

${ }^{1}$ Observatorio Astronomico Nacional SPM, Instituto de Astronomia, UNAM, Ensenada, BC, Mexico e-mail: zhar@astrosen.unam.mx

2 Ioffe Physical Technical Institute, Politekhnicheskaya 26, St. Petersburg, 194021, Russia e-mail: kopts@astro.ioffe.rssi.ru; shib@stella.ioffe.rssi.ru

3 Department of Physics, Tokyo Institute of Technology, 2-12-1 Ookayama, Meguro-ku, Tokyo 152-8551, Japan

${ }^{4}$ RIKEN (Institute of Physical and Chemical Research), 2-1 Hirosawa, Wako, Saitama 351-0198, Japan e-mail: nkawai@hp.phys.titech.ac.jp, urata@crab.riken.go.jp

5 Special Astrophysical Observatory of RAS, Karachai-Cherkessia, Nizhnij Arkhyz, 357167, Russia

${ }^{6}$ Isaac Newton Institute of Chile, SAO Branch, Russia e-mail: vkom@sao.ru, sokolov@sao.ru

7 Department of Physics, Yamagata University, Yamagata 990-8560, Japan e-mail: shibata@sci.kj.yamagata-u.ac.jp

8 Department of Physics, Rikkyo University, Nishi-Ikebukuro, Tokyo 171-8501, Japan e-mail: shibazak@rikkyo.ac.jp

Received 14 June 2002 / Accepted 8 August 2002

\begin{abstract}
We report the $B$ band optical observations of an old ( $\tau \sim 17.5 \mathrm{Myr}$ ) radiopulsar PSR B0950+08 obtained with the Suprime-Cam at the Subaru telescope. We detected a faint object, $B=27.07 \pm 0.16$. Within our astrometrical accuracy it coincides with the radio position of the pulsar and with the object detected earlier by Pavlov et al. (1996) in UV with the $H S T / F O C / F 130 L P$. The positional coincidence and spectral properties of the object suggest that it is the optical counterpart of PSR B0950+08. Its flux in the $B$ band is two times higher than one would expect from the suggested earlier Rayleigh-Jeans interpretation of the only available HST observations in the adjacent F130LP band. Based on the $B$ and F130LP photometry of the suggested counterpart and on the available X-ray data we argue in favour of nonthermal origin of the broad-band optical spectrum of PSR B0950+08, as it is observed for the optical emission of the younger, middle-aged pulsars PSR B0656+14 and Geminga. At the same time, the optical efficiency of PSR B0950+08, estimated from its spin-down power and the detected optical flux, is by several orders of magnitude higher than for these pulsars, and comparable with that for the much younger and more energetic Crab pulsar. We cannot exclude the presence of a compact, $\sim 1$ ", faint pulsar nebula around PSR B0950+08, elongated perpendicular to the vector of its proper motion, unless it is not a projection of a faint extended object on the pulsar position.
\end{abstract}

Key words. pulsars: general - pulsars: individual: PSR B0950+08 - stars: neutron

\section{Introduction}

PSR B0950+08 is the fourth in the list of the first radio pulsars discovered in 1968 (e.g., Bell Burnell 1998). Since that time it has been extensively studied in a wide radio frequency range, from 0.102 to $10.55 \mathrm{GHz}$. The pulsar parameters (period $P$ and its derivative $\dot{P}$, age $\tau$, magnetic field $B$, dispersion measure $D M$, spin-down luminosity $\dot{E}$, distance $d$, position $\alpha, \delta$, proper motion $\mu_{\alpha}, \mu_{\delta}$, and parallax $\pi$ ) are shown in Table 1 . So far this is the oldest pulsar among ordinary pulsars detected outside the radio range.

Send offprint requests to: Yu. A. Shibanov,

e-mail: shib@stella.ioffe.rssi.ru

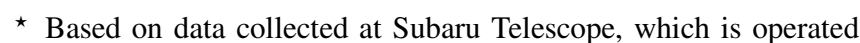
by the National Astronomical Observatory of Japan.
The X-ray counterpart of PSR B0950+08 was first found by Seward \& Wang (1988) and Cordova et al. (1989) with the Einstein observatory. Later, it was observed with the ROSAT by Manning \& Willmore (1994), who analyzed the soft X-ray spectrum in (0.08-2.4) keV range. The blackbody (hereafter BB) spectral fit gave a temperature $T_{\mathrm{BB}}=(2.1 \pm 0.6) \times 10^{6} \mathrm{~K}$ ( $90 \%$ confidence limits) and a very small radius of the emitting region $R_{\mathrm{BB}} \leq 20 \mathrm{~m}$. The pulsar distance was assumed to be of about $130 \mathrm{pc}$ based on early radio parallax measurements by Gwinn et al. (1986) with the VLBI. The power law (hereafter PL, $F_{v} \propto v^{-\alpha_{v}}$ ) spectral fit was also acceptable, but, owing to the poor count statistics for this faint X-ray object, it resulted in a very uncertain spectral index $-0.2 \leq \alpha_{v} \leq 3.1$. An evidence of the pulsed X-ray emission with the pulsar period was reported by Wang \& Halpern (1997) using archival 
Table 1. Parameters of PSR B0950+08 (from Taylor et al. 1993, unless specified otherwise).

\begin{tabular}{|c|c|c|c|c|c|c|c|c|c|}
\hline \multicolumn{6}{|c|}{ Observed } & \multicolumn{4}{|c|}{ Derived } \\
\hline $\begin{array}{c}P \\
\mathrm{~ms} \\
\end{array}$ & $\begin{array}{c}\dot{P} \\
10^{-15} \\
\end{array}$ & $\begin{array}{c}D M \\
\mathrm{~cm}^{-3} \mathrm{pc} \\
\end{array}$ & $\alpha_{2000}, \delta_{2000}^{a, b}$ & $\begin{array}{c}\mu_{\alpha}, \mu_{\delta}^{b} \\
\operatorname{mas}_{\mathrm{yr}^{-1}}\end{array}$ & $\begin{array}{c}\pi^{b} \\
\text { mas } \\
\end{array}$ & $\begin{array}{c}\tau \\
\mathrm{Myr} \\
\end{array}$ & $\begin{array}{l}B \\
\mathrm{G} \\
\end{array}$ & $\begin{array}{c}\dot{E} \\
\operatorname{erg} s^{-1} \\
\end{array}$ & $\begin{array}{r}d^{b, c} \\
\mathrm{pc} \\
\end{array}$ \\
\hline 253 & 0.229 & 2.97 & $\begin{array}{l}9^{\mathrm{h}} 53^{\mathrm{m}} 09^{\mathrm{s}} 313(3)^{d} \\
7^{\circ} 55^{\prime} 36^{\prime \prime} 08(4)\end{array}$ & $\begin{array}{l}-2.09(8) \\
29.46(7)\end{array}$ & $3.82(7)$ & 17.5 & $0.24 \times 10^{12}$ & $5.6 \times 10^{32}$ & $262(5)$ \\
\hline $\begin{array}{l}{ }^{a} \mathrm{Th} \\
{ }^{b} \mathrm{Se} \\
{ }^{c} \mathrm{~Pa}\end{array}$ & $\begin{array}{l}\text { positior } \\
\text { Brisken } \\
\text { llax bas }\end{array}$ & $\begin{array}{l}\text { at the epo } \\
\text { al. (200 } \\
\text { distance }\end{array}$ & the Subaru of & ions, 1 & & $\begin{array}{r}{ }^{d} \text { The num } \\
\text { referring } \\
\text { e.g., } 1.23\end{array}$ & $\begin{array}{l}\text { tors in parenth } \\
\text { to the last sig } \\
4(56)=1.234\end{array}$ & $\begin{array}{l}\text { ses are un } \\
\text { ificant dig } \\
\pm 0.056\end{array}$ & $\begin{array}{l}\text { tainties } \\
\text { uoted, }\end{array}$ \\
\hline
\end{tabular}

ASCA observations in (0.5-5) keV range. However, later the authors recalled their ASCA measurements of PSR B0950+08 since they discovered that the pulsar flux in ASCA data is heavily contaminated by a brighter X-ray source, a previously unknown Seyfert galaxy, only 1.5 away from the pulsar (see a short Note in Wang et al. 1998). In accordance with NS cooling theories, the temperature of the whole surface of this relatively old NS should be less than $10^{5} \mathrm{~K}$ (e.g., Yakovlev et al. 1999). This is much less than observed by the ROSAT. The high temperature and small emitting area inferred from the BB fit can be explained by the presence of small hot polar caps at the NS surface produced by the impact of relativistic particles from the pulsar magnetosphere, see, e.g., a discussion in Wang \& Halpern (1997) and references therein.

An optical counterpart of PSR B0950+08 was suggested by Pavlov et al. (1996) based on observations of the pulsar field with the HST/FOC with the long-pass F130LP filter $(\lambda \lambda=2310-4530 \AA)$. A faint $\left(m_{\mathrm{F} 130 \mathrm{LP}}=27 \mathrm{~m} 1\right)$ point-like object was found with the 1 .' 85 offset from the pulsar radio position. The offset was later revised and decreased to $\simeq 1^{\prime \prime}$ by Pavlov (2000). If this is a pulsar, it is the faintest pulsar ever detected in the optical. For comparison, the visual magnitude of the young Crab pulsar, which is about ten times more distant but $1.75 \times 10^{4}$ times younger, is $16^{\mathrm{m}} 65$ (e.g., see the review by Mignani et al. 2000).

Assuming that the detected optical object is the pulsar, Pavlov et al. (1996) showed that the extension of the ROSAT $\mathrm{BB}$ fit into the UV-optical range gives a flux lower than observed by 3-4 orders of magnitude. This excludes thermal radiation from the pulsar polar caps as a possible source of the optical radiation. The assumption that the detected optical flux is due to thermal emission from the entire surface of a NS with a $\mathrm{BB}$ radius $R_{\mathrm{BB}}=13 \mathrm{~km}$ yielded the surface temperature $T_{\mathrm{BB}} \sim 7 \times 10^{4} \mathrm{~K}$ at $\mathrm{d}=130 \mathrm{pc}$. This is still too high for the $\sim 1.75 \times 10^{7}$ yr cooling NS and can be only explained by some reheating mechanism operating inside the star (e.g., Miralles et al. 1998). On the other hand, the optical flux would be in agreement with the extension of the PL X-ray fit if the index $\alpha_{v}$ lies within the $0.26-0.35$ range. It is in agreement with the ROSAT data but needs a confirmation by deeper observations in X-rays and by the detection of the counterpart in other optical bands.

Possible detection of PSR B0950+08 in the $R$ band with the $6 \mathrm{~m}$ telescope BTA has been reported by Sokolov et al. (1998) and Kurt et al. (2000). An object with $R=25.4 \pm 0.3$ was marginally $(S / N \simeq 3$ ) detected in poor seeing conditions.
If confirmed, this detection suggests that the pulsar may be much brighter in the optical and may have a very steep increase of the spectrum towards longer wavelengths than one would expect from the detection in the near UV.

In this paper we report the observations of the PSR B0950+08 field in the $B$ band with the Subaru telescope. We analyze our data together with the available optical-UV data from the HST, and with the X-ray data from the ROSAT, making use of the recent much more accurate radio measurements of the pulsar proper motion, parallax, and distance with the VLBA by Brisken et al. (2002). Observations and data reduction are described in Sect. 2. In Sect. 3 we present the astrometrical referencing and photometry, and in Sect. 4 we discuss the results and their implications.

\section{Observations and data reduction}

The field of PSR B0950+08 was observed on Jan. 21, 2001 with the wide field camera Suprime-Cam at the primary focus of the Subaru telescope. The Suprime-Cam (Miyazaki et al. 1998 ) is equipped with ten MIT/LL $2048 \times 4096$ CCDs arranged in a $5 \times 2$ pattern to provide $34^{\prime} \times 27^{\prime}$ FOV with a pixel size on the sky of $0.201^{\prime \prime} \times 0.201^{\prime \prime}$. The pulsar was exposed in one of the CCD chips, si006s, with the gain $=1.17$. Nine $600 \mathrm{~s}$ exposures were taken using the $B$ filter with the bandpass close to the Johnson system. The mean seeing was about $0.7^{\prime \prime}$. The 2nd, 7th, and 9th exposures were removed from further consideration because of the problems with the telescope guiding system. The observational conditions for the rest six exposures are listed in Table 2.

The standard data reduction, including bias subtraction and flatfielding, was performed making use of the ccdproc task of the IRAF software. To combine the individual dithered images and to get rid of cosmic rays, as well as of a trace of an unknown minor object or a dust clump with an orbit close to the

Table 2. Log of observations of the pulsar in the $B$ band.

\begin{tabular}{ccccc}
\hline \hline $\begin{array}{c}\text { Exposure } \\
\text { number }\end{array}$ & $\begin{array}{c}\text { UT } \\
\text { 21 Jan. 2001 }\end{array}$ & $\begin{array}{c}\text { Duration } \\
\text { s }\end{array}$ & Airmass & $\begin{array}{c}\text { Seeing } \\
\text { arcsec }\end{array}$ \\
\hline 1 & $12: 41$ & 600 & 1.037 & 0.65 \\
3 & $13: 21$ & 600 & 1.085 & 0.66 \\
4 & $13: 54$ & 600 & 1.129 & 0.69 \\
5 & $14: 08$ & 600 & 1.164 & 0.72 \\
6 & $14: 22$ & 600 & 1.205 & 0.73 \\
8 & $14: 50$ & 600 & 1.313 & 0.75 \\
\hline
\end{tabular}



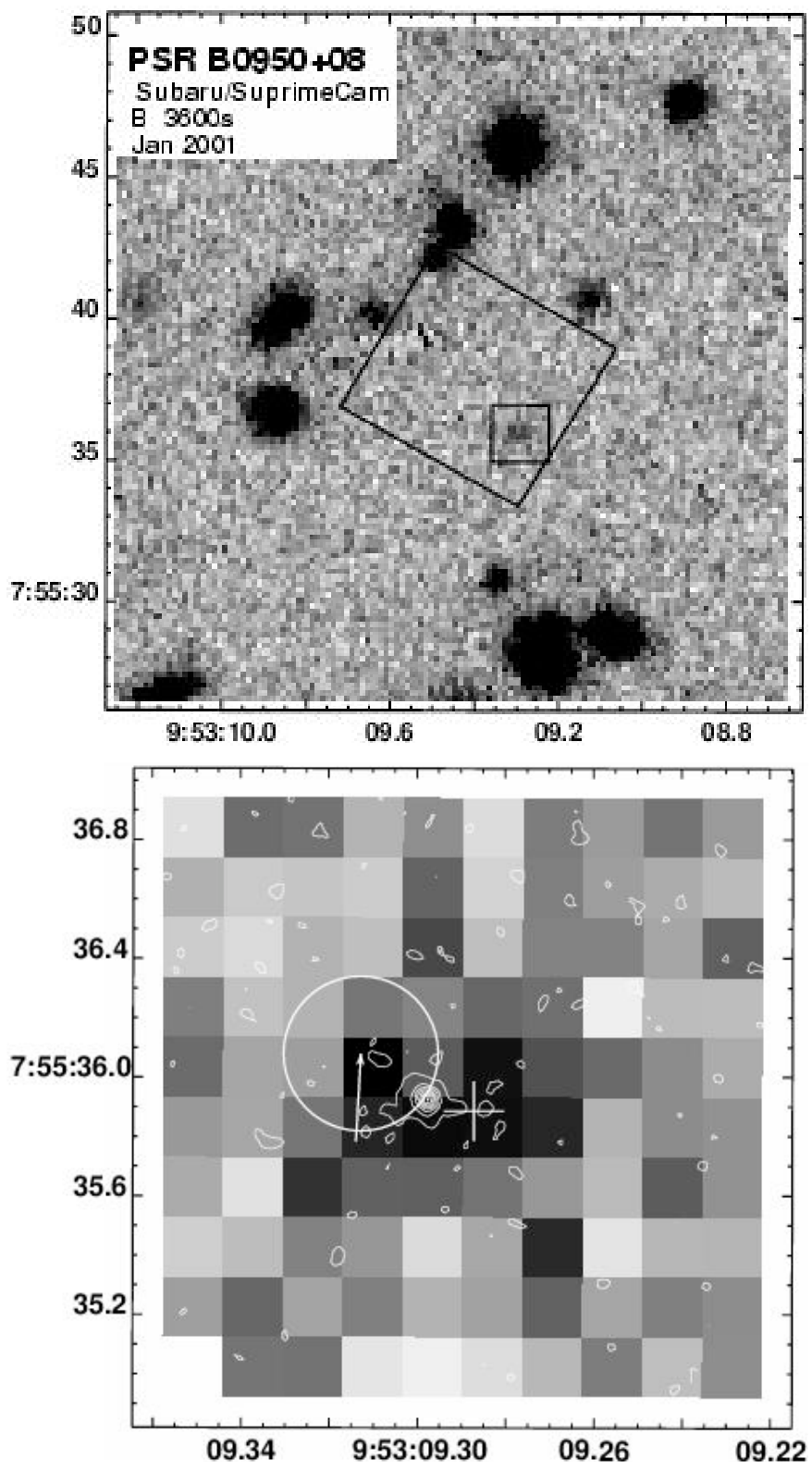

Earth, crossing our image E-W approximately $8^{\prime \prime}$ away of the pulsar position, we made use of the IRAF dither package. A fragment of the combined image of the pulsar field with the total integrated exposure time of $3600 \mathrm{~s}$ is shown in Fig. $1 \mathrm{a}^{1}$.

\section{Astrometrical referencing and photometry}

\subsection{Astrometry}

The radio position of PSR B0950+08 at the epoch of the observations (see Table 1) was determined using the VLA observations by Fomalont et al. (1992) and the most recent measurements of the pulsar proper motion by Brisken et al. (2002).

\footnotetext{
1 Image is available in FITS format at http://www .ioffe.rssi.ru/astro/NSG/obs/0950-subaru
}

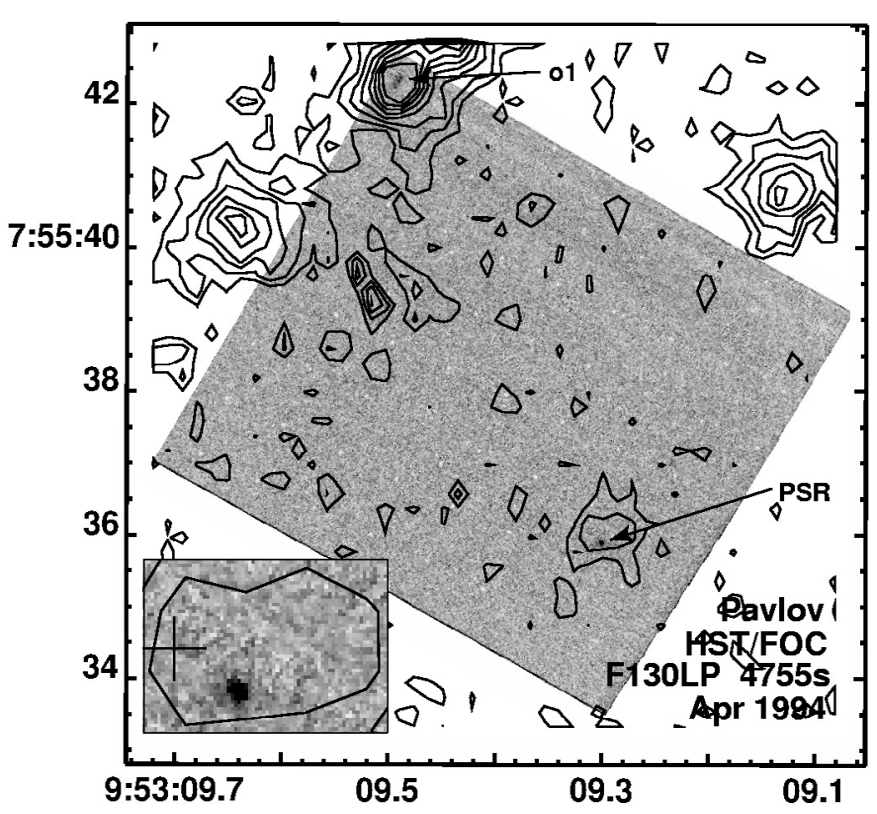

Fig. 1. Top Left a) $24^{\prime \prime} \times 24^{\prime \prime}$ fragment of the Subaru $2001 \mathrm{im}-$ age of the PSR B0950+08 field in the $B$ band. Larger and smaller boxes show the FOV of the HST 1994 observations (see the right panel) and the borders of the bottom panel containing the pulsar, respectively. Right b) 7'.4 × 7'.4 HST 1994 image of the pulsar field. The pulsar counterpart and an extended object o1, both visible in the HST and Subaru images, are marked. The HST image is aligned with the Subaru image using o1 as a reference. The Subaru contour map is overlaid. The pulsar close vicinity, $\sim 0{ }^{\prime} 9 \times 0$.' 6 , with the closest Subaru contour and the radioposition at the epoch of the FOC observations marked by "+", is zoomed in the inset. Bottom c) $2^{\prime \prime} \times 2^{\prime \prime}$ neighbourhood of the pulsar in the $B$ band. The HST contour map is overlaid. It is shifted for the pulsar proper motion for $6.75 \mathrm{yr}$ passed between the observations. The arrow points out the radio position of the pulsar at the epoch of the Subaru observations, its length corresponds to the pulsar shift due to the proper motion for $10 \mathrm{yr}$. The circle shows $1 \sigma$ uncertainty of the pulsar position in the Subaru image; "+" marks the center of the Gaussian fitted to the pulsar counterpart profile in this image.

Astrometric referencing of our image was made using positions of five ${ }^{2}$ of nine reference stars from the USNO A-2.0 catalogue visible within the $\sim 6^{\prime} \times 13^{\prime}$ frame of the CCD chip containing the pulsar. The PSFs of these stars are not corrupted by the CCD oversaturation effects. We used IRAF tasks ccmap/cctran for the astrometric transformation of the image. RMS errors of the astrometric fit are 0.'086 and 0.'090 for RA and Dec, respectively, whereas the residuals for all stars are $<0$.' 13 . All of them are less than the nominal USNO catalogue accuracy 0!'24. Combining the RMS errors of the fit, the USNO catalogue accuracy and the radio position errors (see Table 1) we estimate total uncertainties of the pulsar position, as well

\footnotetext{
2 U0975_06358239, U0975_06357626, U0975_06357575, U0975_06358752, U0975_06358583.
} 
as the astrometric referencing accuracy for the whole Subaru image, as 0.26 in both RA and $\mathrm{Dec}^{3}$.

\subsection{Identification and morphology of the candidate to the pulsar counterpart}

As seen from Fig. 1, the optical counterpart of PSR B0950+08 can only be identified with a faint, $S / N \simeq 7$, isolated object clearly visible near the center of the $24^{\prime \prime} \times 24^{\prime \prime}$ fragment of the Subaru image (Fig. 1a). The object overlaps with the error circle of the pulsar radio position with radius 0 '.26, corresponding to $1 \sigma$ uncertainty of our astrometrical referencing (Fig. 1c). The source profile of the object is elongated E-W, with $F W H M s \sim 11^{\prime \prime} 0$ and $\sim 0$ ' 7 along E-W and S-N directions, respectively. Owing to the low $S / N$ ratio, we do not resolve any point-like structure within the source profile. Formal Gaussian fitting of the whole object profile yields the coordinates $\alpha_{2000}=9^{\mathrm{h}} 53^{\mathrm{m}} 09^{\mathrm{s}} \mathrm{s} 288(17), \delta_{2000}=7^{\circ} 55^{\prime} 35^{\prime \prime} \cdot 89(26)$. The offset from the radio position is 0 ! $^{\prime} 43 \mathrm{~W}-\mathrm{S}$. It is within $1.75 \sigma$ of the astrometrical accuracy and can be considered as negligible given our seeing and $S / N$ values.

To compare our image with the previous optical-UV observations of the pulsar field by Pavlov et al. (1996) we reanalyzed astrometrical referencing of the HST/FOC image retrieved from the HST archive. The rotated box in Fig. 1a borders the FOV of the HST/FOC observations. Pavlov et al. (1996) found in this field the only point-like object with the offset $\simeq 1^{\prime \prime}$ (Pavlov 2000) from the pulsar radio position. Such a large offset at the relatively high spatial resolution of the HST/FOC observations, $\simeq 0$ ' 014 , makes identification of the FOC object with the pulsar and with the Subaru pulsar counterpart doubtful. We revised the FOC astrometry making use of the FOC position angle and the only reference point visible at the north corner of the FOC image, an extended object o1 (see Fig. 1b). We assumed that $\mathrm{o} 1$ is a distant background object and its proper motion is negligible. Gaussian center coordinates of o1 were determined in the Subaru image $\left(\alpha_{2000}=9^{\mathrm{h}} 53^{\mathrm{m}} 09^{\mathrm{s}} .489(2)\right.$, $\delta_{2000}=7^{\circ} 55^{\prime} 42^{\prime \prime} 2(1)$ ) and in the FOC image (with the accuracy $\lessgtr 00^{\prime} 02$ ), and were used to correct reference frame of the FOC image. The coordinates of the FOC pulsar candidate in the corrected system at the epoch of the FOC observations are $\alpha_{2000}=9^{\mathrm{h}} 53^{\mathrm{m}} 09^{\mathrm{s}} .298, \delta_{2000}=7^{\circ} 55^{\prime} 35^{\prime \prime}$.76. Given that, the discrepancy between the FOC counterpart position and the radio position at the epoch of the HST observations, $\alpha_{2000}=9^{\mathrm{h}} 53^{\mathrm{m}} 09^{\mathrm{s}} .314(3), \delta_{2000}=7^{\circ} 55^{\prime} 35^{\prime \prime} .88(4)$, marked by "+" in the inset in Fig. 1b, is decreased to 0!'27. This is comparable to the astrometrical referencing accuracy of the Subaru image.

The FOC image is presented in Fig. $1 \mathrm{~b}$ in the corrected coordinates. The overlaid contour map of the Subaru image shows that the Subaru and HST detected the same object near the pulsar position. In the blown up image of the object in Fig. 1c the FOC contour map is additionally shifted with respect to the Subaru image by $-0{ }^{\prime} 014$ in RA and $0{ }^{\prime} 199$ in Dec to compensate for the pulsar proper motion during the $6.75 \mathrm{yr}$ interval

\footnotetext{
3 There are no reference stars south of the pulsar within our frame and thus we cannot exclude a systematic shift in the $\mathrm{N}-\mathrm{S}$ direction.
}

between the HST and Subaru observations. Isophotes on the contour maps in Figs. 1b,c correspond to the levels (in counts) above the background $l_{n}=S+n \sigma$, where $S$ is the mean sky value near the pulsar, $\sigma$ is the sky standard deviation related to one pixel, $n=1,2,3, \ldots, 8$ and $1,3,5, \ldots, 15$ for the Subaru and HST maps, respectively. The better agreement of the Subaru and HST source positions after the correction for the pulsar proper motion in Fig. 1c favours the object as the optical counterpart of PSR B0950+08.

We also note that, although the HST/FOC pulsar counterpart profile is point-like, the edges of its wings are slightly elongated E-W (Fig. 1c). The elongation directions in both the FOC and Subaru images coincide and are approximately orthogonal to the vector of the proper motion of the pulsar marked by the arrow in Fig. 1c. Such an orientation may suggest the association of the elongation with a faint torus-like structure of a possible compact pulsar nebula as detected around young Crab-like pulsars (e.g., Weisskopf et al. 2000). However, it may be also a projection of a faint extended background object at the pulsar position.

To summarize, our analysis shows that the HST and Subaru detected the same object. With the allowance for the pulsar proper motion, the offsets from the PSR B0950+08 radio positions at the HST and Subaru observational epochs are in the range (0.'3-0.'4), which are within $1.7 \sigma$ error of the Subaru astrometrical referencing and negligible compared to our seeing of 0.7 .

\subsection{Photometry}

Weather conditions were stable during our observations. We derived the atmospheric extinction coefficient in the $B$ band $k_{B}=0.18 \pm 0.02$ from the variation of the count rates of four stars in the pulsar field with airmass during our observations (see Table 2). Insignificant decrease of the extinction, within 1- $\sigma$ level, was noticed from the beginning to the end of observations.

Photometric referencing was carried out using three defocused standard stars from the field PG1047 (Landolt 1992) with $V \sim 13$, along with five fainter, $V \sim 21$, unsaturated secondary standard stars from the PSR B0656+14 field (Kurt et al. 1998) observed in the BRI bands the same night. The derived photometric zeropoint in the $B$ band was $28.28 \pm 0.02$. The instrumental magnitudes of the detected optical source were measured for a range of aperture radii of (1-3) CCD pixels centered at the "+" in Fig. 1c. They were corrected for the PSF of bright stars (some details on the method we used can be found in Koptsevich et al. 2001). Within the measurement errors, the results for different apertures coincided and a 2 pixel radius was adopted as optimal. The magnitude of the detected optical source, $B=27.07 \pm 0.16$, corresponds to the absolute flux $F_{B}=(5.97 \pm 0.88) \times 10^{-31} \mathrm{erg} \mathrm{cm}^{-2} \mathrm{~s}^{-1} \mathrm{~Hz}^{-1}$ or $0.0597 \pm 0.0088 \mu \mathrm{Jy}$. The error includes statistical error of the instrumental magnitude measurements, the error of the zeropoint, and an allowance for possible atmospheric extinction variations. 


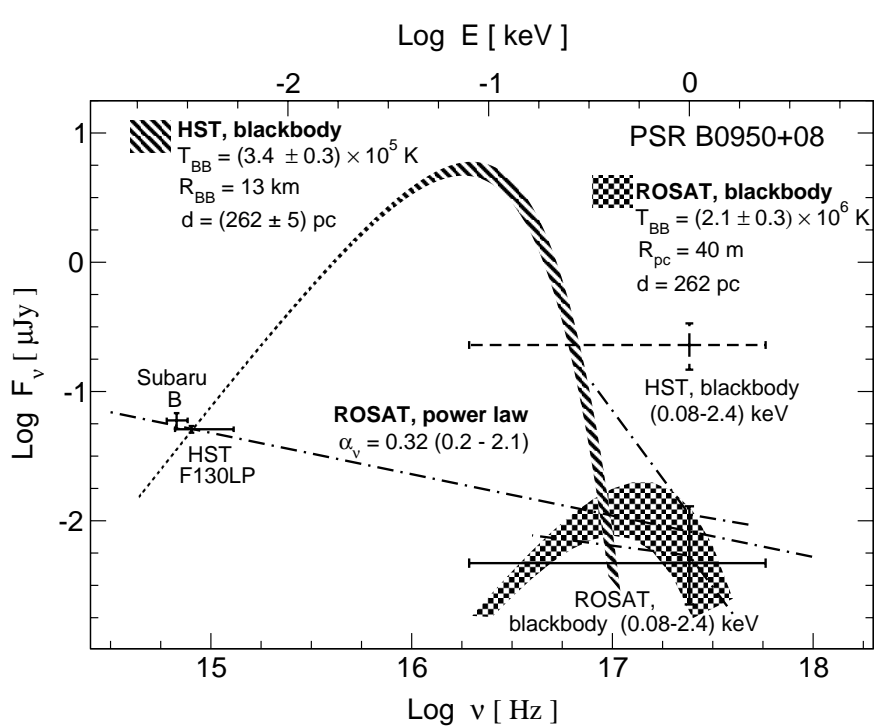

Fig. 2. Optical and X-ray observations of PSR B0950+08. Solid cross in the X-ray range corresponds to the mean flux in the $E=(0.08-$ 2.4) $\mathrm{keV}$ band derived from the BB spectral fit of the ROSAT data (Manning \& Willmore 1994). Square-filled belt shows unabsorbed spectrum resulted from this fit. Dot-dashes outline the results of the PL fit of the same data with $\pm 1 \sigma$ uncertainties. Stripe-filled belt shows unabsorbed BB fit of the HST/FOC data, and dashed cross shows corresponding mean flux as one would expect in the ROSAT band. The fits are extrapolated toward the X-ray and optical ranges, respectively, and their parameters are shown in the plot. The widths of the belts correspond to $\pm 1 \sigma$ uncertainties of the fits. As seen, the optical emission is likely to be of nonthermal origin and apparently follows the extrapolation of the X-ray PL fit of the ROSAT data with a spectral index $\alpha_{v} \simeq 0.32$ close to the low boundary of $1 \sigma$ uncertainty of the fit (see Sect. 4 for details).

\section{Discussion}

In Fig. 2 we combine our results with the available data from the optical and X-ray observations of PSR B0950+08. In the optical range we neglect the interstellar extinction, which is expected to be very low for this pulsar, $E_{B-V} \lesssim 0.02$ (e.g., Pavlov et al. 1996). We excluded from our consideration the observations in the $R$ band by Kurt et al. (2000) because of very low signal-to-noise ratio. In the $\mathrm{X}$-ray range we show unabsorbed fluxes resulted from the BB and PL spectral fits of the ROSAT (Manning \& Willmore 1994) data. The fits are rescaled to the new distance value $d=262 \pm 5$ pc measured by Brisken et al. (2002).

Within the errors the flux $F_{\mathrm{B}}$ coincides with the value $F_{\mathrm{F} 130 \mathrm{LP}}=0.051 \pm 0.003 \mu \mathrm{Jy}$ measured by Pavlov et al. (1996) with the HST/FOC in the F130LP band. The $B$ band $(\lambda \lambda \sim$ 3944-4952 $\AA$ ) considerably overlaps with the F130LP band ( $\lambda \lambda \sim 2310-4530 \AA)$. However, their pivot wavelengths $\lambda_{B}^{0}=$ $4448 \AA$ and $\lambda_{\mathrm{F} 130 \mathrm{LP}}^{0}=3750 \AA$ are different. This difference and close flux values in two bands suggest a flat spectrum of the object in a wide $\lambda \lambda \sim 2300-5000 \AA$ spectral range. Along with the positional coincidence, we consider this flat spectrum, which is typical for pulsar optical spectra (e.g., Koptsevich et al. 2001; Mignani \& Caraveo 2001), as an additional argument in favour of the detection of the PSR B0950+08 optical counterpart.
If the detected object is the pulsar, its flux in the $B$ band does not follow the Rayleigh-Jeans law suggested by Pavlov et al. (1996) to explain the optical-UV radiation in the only F130LP band as a low temperature thermal emission from the entire surface of a reheating/cooling NS with $R_{\infty}=13 \mathrm{~km}$. In that case in the $B$ band we would detect twice smaller flux than measured. This is well outside the uncertainties and suggests a nonthermal origin of the pulsar emission at least in the $B$ band.

If the flux in the F130LP band is still dominated by the Rayleigh-Jeans tail, we would obtain $T_{\mathrm{BB}}=(3.4 \pm 0.3) \times$ $10^{5} \mathrm{~K}$, which is a factor of 4-5 higher than found by Pavlov et al. (1996), mainly owing to the change of the distance to PSR B0950+08 from 130 pc to 262 pc (see the stripe-filled BB belt crossing the F130LP band and extended to X-rays in Fig. 2). A NS with such a hot surface would produce a flux $\sim(0.8-1.9) \times 10^{-12} \mathrm{erg} \mathrm{cm}^{-2} \mathrm{~s}^{-1}$ in the $(0.08-2.4) \mathrm{keV}$ band (see a big dashed cross in Fig. 2). It is well above the value $(2.4-7.3) \times 10^{-14} \mathrm{erg} \mathrm{cm}^{-2} \mathrm{~s}^{-1}$, measured by Manning $\&$ Willmore (1994) with the ROSAT under the assumption of the BB spectrum of the detected X-ray radiation (marked by a big cross below the dashed one in Fig. 2). This means that the whole surface of PSR B0950+08 is actually much cooler and its emission cannot dominate in the F130LP band. Thermal emission from hotter, $T_{\mathrm{BB}}=(2.1 \pm 0.3) \times 10^{6} \mathrm{~K}$, but much smaller polar caps of the pulsar with $R_{\mathrm{pc}} \sim 40 \mathrm{~m}$, which may explain the detected $\mathrm{X}$-ray radiation, can hardly be visible in the optical range also because of very small areas of the caps inferred from the BB fit of the X-ray data (see square-filled BB belt in Fig. 2).

For the above reasons it is most likely that the optical radiation of PSR B0950+08 is completely dominated by nonthermal emission produced in the magnetosphere of the rapidly rotating NS, as it is believed to be for young and well studied pulsars like the Crab and PSR B0540-69. Within large uncertainties of the available X-ray data for PSR B0950+08 the PL with the spectral index $\alpha_{v} \simeq 0.32$ matches both the X-ray and optical fluxes including the $B$ and F130LP bands (straight dot-dashed line in Fig. 2). This value is consistent with what was obtained by Manning \& Willmore (1994) from the analysis of the ROSAT X-ray data: $F_{\mathrm{ROSAT}}^{\mathrm{PL}}=6.3(+12.3,-0.34) \times$

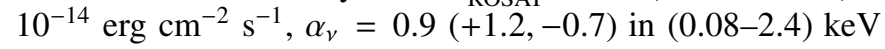
energy range at $68 \%$ confidence levels (see dot-dashed broken lines in Fig. 2). The PL fit of the X-ray data is more preferable since the BB fit, which is statistically also acceptable, implies by an order of magnitude smaller emitting area than it is predicted by standard models of hot polar caps at the pulsar surface (e.g., Arons 1981). The inferred spectral index differs from $\alpha_{v} \sim 1$ which is typical for nonthermal soft X-ray radiation of most rotation-powered NSs (e.g., Becker \& Trümper 1997). However, it can be as low as 0.4 for middle-aged pulsars (Koptsevich et al. 2001) and we cannot exclude a decrease of the slope of the PSR B0950+08 spectrum towards the optical range as it is seen in the case of the Crab pulsar (e.g., CrusiusWätzel et al. 2001). More X-ray and optical data are needed to check the spectral shape for PSR B0950+08. With new data it would be also useful to perform the BB + PL, and/or NS atmosphere + PL fits (e.g., Zavlin et al. 1996) to better constrain the parameters of the nonthermal and thermal spectral components 
Table 3. Parameters of the radio pulsars and the efficiencies of their optical emission $\eta_{B}$ in the $B$ band.

\begin{tabular}{lccccccc}
\hline \hline Pulsar & $\begin{array}{c}\log \tau \\
\mathrm{yr}\end{array}$ & $\begin{array}{c}d \\
\mathrm{kpc}\end{array}$ & $\begin{array}{c}\log \dot{E} \\
\mathrm{erg} \mathrm{s}^{-1}\end{array}$ & $\begin{array}{c}B \\
\mathrm{mag}\end{array}$ & $\begin{array}{c}M_{B} \\
\mathrm{mag}\end{array}$ & $\begin{array}{c}\log L_{B} \\
\mathrm{erg} \mathrm{s}^{-1}\end{array}$ & $\log \eta_{B}$ \\
\hline Crab & 3.1 & $2.0(1)$ & 38.65 & $15.25(7)^{1}$ & $3.74(13)$ & $33.23(5)$ & $-5.42(5)$ \\
B0540-69 & 3.2 & $50_{-0.6}^{+5}$ & 38.17 & $22.0(3)^{2}$ & $3.48(37)$ & $33.47(15)$ & $-4.7(2)$ \\
Vela & 4.1 & $0.294_{-0.050}^{+0.070^{a}}$ & 36.84 & $\left.23.7^{3} 3\right)^{3}$ & $16.4_{-0.8}^{+0.5}$ & $28.3(3)$ & $-8.5(3)$ \\
B0656+14 & 5.0 & $0.5_{-0.3}^{+0.26^{b}}$ & 34.58 & $25.15(13)^{4}$ & $16.8_{-1.0}^{+2.1}$ & $28.2_{-0.9}^{+0.4}$ & $-6.4_{-0.9}^{+0.4}$ \\
Geminga & 5.5 & $0.153_{-0.034}^{+0.059}$ & 34.51 & $25.7(3)^{5}$ & $19.8_{-1.0}^{+0.8}$ & $26.95_{-0.10}^{+0.16}$ & $-7.56_{-0.10}^{+0.16}$ \\
B1929+10 & 6.5 & $0.331(10)^{d}$ & 33.59 & $\geq 26.2^{6}$ & $20.0_{-0.2}^{+0.2^{* *}}$ & $27.26_{-0.3}^{+0.2^{* *}}$ & $-6.3_{-0.3}^{+0.2^{* *}}$ \\
B0950+08 & 7.2 & $0.262(5)^{d}$ & 32.75 & $27.07(16)^{7}$ & $19.98(19)$ & $26.88(8)$ & $-5.87(8)$ \\
\hline
\end{tabular}

The $B$ band magnitudes are from:

${ }^{1}$ Percival et al. (1993); ${ }^{2}$ Middleditch et al. (1987) (spectroscopic data by Hill et al. (1997) give $\sim 1.5$ smaller magnitude); ${ }^{3}$ Nasuti et al. (1997);

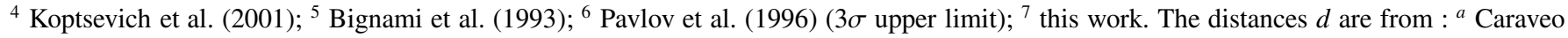
et al. (2001); ${ }^{b}$ Taylor et al. (1993), Finley et al. (1992), Anderson et al. (1993); ${ }^{c}$ Caraveo et al. (1996); ${ }^{d}$ Brisken et al. (2002). ${ }^{* *}$ The estimates are based on the counterpart detection in the adjacent F130LP band by Pavlov et al. (1996), see Sect. 4.

Uncertainties of $M_{B}, L_{B}$ and $\eta_{B}$ include uncertainties of the optical flux and distance measurements.

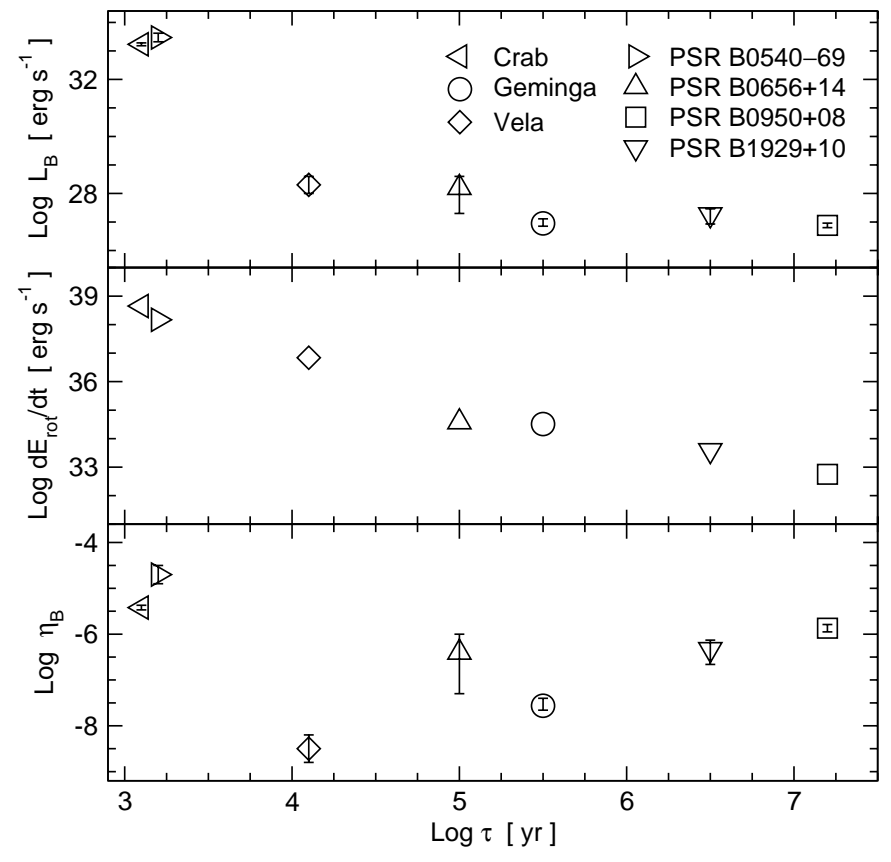

Fig. 3. The optical luminosity $L_{B}$ (top), spin-down power $\dot{E}$ (middle) and optical efficiency in the $B$ band $\eta_{B}$ (bottom) for all radio pulsars detected in the optical range as functions of pulsar age $\tau$ (see also Table 3).

from the pulsar magnetosphere and polar caps and to estimate their contribution to the pulsar emission in different spectral bands.

If the detected object is PSR B0950+08, the pulsar optical luminosity in the $B$ band, assuming isotropy of the emission, is $L_{B}=4 \pi d^{2} F_{v} \Delta v=(7.6 \pm 1.2) \times 10^{26} d_{262}^{2} \mathrm{erg} \mathrm{s}^{-1}$, where $d_{262}=d /(262 \mathrm{pc})$ is the normalized distance. As seen from Table 3 and Fig. 3 (top panel), where we collected the data in the $B$ band for all pulsars detected in the optical range, $L_{B}$ of PSR B0950+08 is comparable with the luminosity of the middle-aged Geminga pulsar but by almost six orders of magnitude smaller than $L_{B}$ of much younger and energetic Crab pulsar. These data indicate that, starting approximately from the age of Geminga, the decrease of the optical luminosity with the pulsar age becomes slower than at early stages of the pulsar evolution or even disappears completely, while the spin-down luminosity continues to decrease with approximately the same rate (Fig. 3, middle panel). This means that for unknown reasons mechanisms of the optical emission may start to be more efficient again after $\sim 10^{6} \mathrm{yr}$ age. The ratio of the optical luminosity to the pulsar spin-down luminosity, $\eta_{B}=L_{B} / \dot{E}$, which can be considered as a rough characteristic of this efficiency, is plotted in Fig. 3 (bottom panel). It is seen that optical photons are produced by the old PSR B0950+08 with almost the same efficiency as by the young Crab pulsar. In this respect it is not surprising that we see some indications of the presence of a compact pulsar nebula around PSR B0950+08. Manning \& Willmore (1994) discuss a compact synchrotron nebula, which is unresolved with the ROSAT, as the most likely explanation of the observed X-ray spectrum of the pulsar. The estimated sizes of the possible X-ray nebula $\sim\left(6^{\prime \prime}-1^{\prime}\right)$ are much larger than $\sim 1^{\prime \prime}$ we see in our optical images. But the difference in the X-ray and optical sizes is typical for known compact pulsar nebulae where only internal brightest structures of a nebula are visible in the optical range (e.g., Weisskopf et al. 2000).

The efficiency derived by Pavlov et al. (1996) in the F130LP band for another old, $\sim 3.1 \times 10^{6} \mathrm{yr}$, PSR B1929+10 is about $(3-8) \times 10^{-7} d_{170}^{2}$. This pulsar has not yet been detected in the adjacent $B$ band, but we can assume that its flux in $B$ is close to that in F130LP, as it is for PSR B0950+08. Scaling by the factor $\Delta v_{\mathrm{B}} / \Delta v_{\mathrm{F} 130 \mathrm{LP}}=0.24$ and by the new radio parallax based distance $d=331$ pc (Brisken et al. 2002) yields $\eta_{B} \sim(2.7-7.3) \times 10^{-7} d_{331}^{2}$. With this value PSR B1929+10 occupies an intermediate position at the rising part of the dependence of $\eta_{B}(\tau)$, between Geminga and PSR B0950+08, as it is expected from its age. Although our consideration of the efficiency evolution is based on the data obtained only in the $B$ band, it appears to be qualitatively valid for the whole optical range since the broad-band spectra of all pulsars are almost flat. Hence, optical luminosities of various pulsars should not be affected strongly by insignificant differences of their spectral slopes in this range. 
Physical reasons for such a high increase of the optical efficiency at late stages of the pulsar evolution are not quite clear. We can only note that the efficiency for gamma-ray radiation, $\eta_{\gamma}=L_{\gamma} / \dot{E}$, also appears to be generally higher for older pulsars (e.g., Thompson 2000). It is difficult to estimate statistical significances of these facts and their possible correlation since the numbers of pulsars currently detected in the optical and gamma-rays are too small (about ten only). Moreover, not all known gamma-ray pulsars are detected in the optical range (and vice versa). However, the observed tendency in both ranges seems to be interesting and can hardly be ignored. It is obvious that in the both wavelength ranges the radiation is nonthermal and originates in magnetospheres of rapidly rotating NSs. Thus, the increase of the efficiencies in two very different ranges may reflect an overall increase of the magnetospheric activity with the NS spin-down. A global electrodynamic model of the pulsar magnetosphere with the activity caused by the magnetic field-aligned potential drop producing electron-positron pairs in the magnetic polar regions of the magnetosphere predicts that the efficiency should increase for high energy photons $\propto P^{2}$ (Shibata 1995). However, the optical data, particularly for young pulsars, do not follow this dependence and detailed studies of the electrodynamics and radiation processes still have to be done to explain the efficiency evolution in different spectral ranges.

Further observations of the candidates to the optical counterparts of the old pulsars PSR B0950+08 and PSR B1929+10 in different spectral bands would be very useful to resolve the efficiency problem and to better understanding the nature of the optical emission of pulsars and the evolution of this emission with pulsar age. Measurements of their proper motion and the detection of pulsations with the pulsar periods in the optical range would be most important to provide firm evidence of the pulsar nature of the detected optical objects. Simultaneous studies of the optical and X-ray pulse profile would provide stronger indications whether the optical and X-ray emissions are generated by the same physical process. Our observations of a very faint PSR B0950+08 with the Subaru show that new generation of large ground-based telescopes is very effective for these studies and could lead to a considerable increase of the number of pulsars detected in the optical range in the near future.

Acknowledgements. This work was supported in part by CONACYT projects 25454-E and 36585-E, RFBR grants 02-02-17668 and 00-07-90183. We are grateful to Y. Komiyama for the help during observations with the Subaru, and to G. Pavlov for the unpublished results on revised astrometrical referencing of the HST/FOC image of the PSR B0950+08 field. Some of the data presented in this paper were obtained from the Multimission Archive at the Space Telescope Science Institute (MAST). STScI is operated by the Association of Universities for Research in Astronomy, Inc., under NASA contract NAS5-26555. Support for MAST for non-HST data is provided by the NASA Office of Space Science via grant NAG5-7584 and by other grants and contracts. ABK is grateful to the Astronomy Department of the University of Washington for hospitality. We are also grateful to M. Richer for careful reading of this text and useful remarks, to V. Palshin for a discussion, and to the referee, J. Halpern, for drawing our attention to the incorrectness of the published ASCA data analysis of PSR B0950+08.

\section{References}

Anderson, S. B., Córdova, F. A., Pavlov, G. G., Robinson, C. R., \& Tompson, R. J. 1993, ApJ, 414, 867

Arons, J. 1981, ApJ, 248, 1099

Becker W., \& Trümper J. 1997, A\&A, 326, 682

Bell Burnell, S. J. 1998, in Neutron Stars and Pulsars, ed. N. Shibazaki, N. Kawai, S. Shibata, \& T. Kifune (Univ. Acad. Press Inc., Tokyo), 1

Bignami, G. F., Caraveo, P. A., \& Mereghetti, S. 1993, Nature, 361, 704

Brisken, W. F., Benson, J. M., Goss, W. M., \& Thorsett, S. E. 2002, ApJ, 571, 906

Caraveo, P. A., Bignami, G. F., Mignani, R., \& Taff, L. G. 1996, ApJ, 461, L91

Caraveo, P. A., De Luca, A., Mignani, R. P., \& Bignami, G. F. 2001, ApJ, 561, 930

Cordova, F. A., Hjellming, R. M., Mason, K. O., \& Middleditch, J. 1989, ApJ, 345, 451

Crusius-Wätzel, A. R., Kunzl, T., \& Lesch, H. 2001, ApJ, 546, 401

Finley, J. P., Ögelman, H., \& Kiziloglu, Ü. 1992, ApJ, 394, L21

Fomalont, E. B., Goss, W. M., Lyne, A. G., Manchester, R. N., \& Justtanont, K. 1992, MNRAS, 258, 497

Gwinn, C. R., Taylor, J. H., Weisberg, J. M., \& Rawley, L. A. 1986, AJ, 91, 338

Hill, R. J., Dolan, J. F., Bless, R. C., et al. 1997, ApJ, 486, L99

Koptsevich, A. B., Pavlov, G. G., Zharikov, S. V., et al. 2001, A\&A, 370, 1004

Kurt, V. G., Sokolov, V. V., Zharikov, S. V., Pavlov, G. G., \& Komberg, B. V. 1998, A\&A, 333, 547

Kurt, V. G., Komarova, V. N., Fatkhullin, T. A., et al. 2000, Bulletin of SAO RAS, 49, 5

Landolt, A. 1992, AJ, 104, 340

Manning, R. A., \& Willmore, A. P. 1994, MNRAS, 266, 635

Middleditch, J., Pennypacker, C. R., \& Burns, M. S. 1987, ApJ, 315, 142

Mignani, R. P., Caraveo, P. A., \& Bignami, G. F. 2000, Msngr, 99, 22

Mignani, R. P., \& Caraveo, P. A. 2001, A\&A, 376, 213

Miyazaki, S., Sekiguchi, M., Imi, K., et al. 1998, SPIE, 3355, 363

Miralles, J. A., Urpin, V., \& Konenkov, D. 1998, ApJ, 503, 368

Nasuti, F. P., Mignani, R., Caraveo, P. A., \& Bignami, G. F. 1997, A\&A, 323, 839

Pavlov, G. G., Stringfellow, G. S., \& Cordova, F. A. 1996, ApJ, 467, 370

Pavlov, G. G. 2000, private communication

Percival, J. W., Biggs, J. D., Dolan, J. F., et al. 1993, ApJ, 407, 276

Seward, F., \& Wang, Z. 1988, ApJ, 332, 199

Shibata, S. 1995, MNRAS, 276, 537

Sokolov, V. V., Kurt, V. G., Zharikov, S. V., Shibanov, Yu. A., \& Koptsevich, A. B. 1998, The 19th Texas Symp. on Relativistic Astrophysics and Cosmology, held in Paris, France, Dec. 14-18, 1998, ed. J. Paul, T. Montmerle, \& E. Aubourg (CEA Saclay)

Taylor, J. H., Manchester, R. N., \& Lyne, A. G. 1993, ApJS, 88, 529.

Thompson, D. 2000, Adv. Space Res., 25, 659

Wang, F.Y.-H., \& Halpern, J. P. 1997, ApJ, 482, L159

Wang, F. Y.-H., Ruderman, M., Halpern, J. P., \& Zhu, T. 1998, ApJ, 498,373

Weisskopf, M. C., Hester, J. J., Tennant, A. F., et al. 2000, ApJ, 536, L81

Yakovlev, D. G., Levenfish, K. P., \& Shibanov, Yu. A. 1999, Physics-Uspekhi, 42, 737

Zavlin, V. E., Pavlov, G. G., \& Shibanov, Yu. A. 1996, A\&A, 315, 141 